\title{
A Novel Bilayer Propionyl-L-carnitine Loaded Polyvinyl Alcohol/Calcium Alginate/Carboxymethyl Cellulose wound dressing for the treatment of diabetic wounds: an in vitro and in vivo study
}

\author{
Wenjun Jiang \\ University of Electronic Science and Technology of China \\ Zuoyao Huang \\ University of Electronic Science and Technology of China \\ Weixiang Min \\ University of Electronic Science and Technology of China \\ Hao Zhang ( $D$ haozhang2021@gmail.com ) \\ University of Electronic Science and Technology of China
}

\section{Research Article}

Keywords: Diabetic wounds, Wound dressing, Propionyl-L-carnitine, Carboxymethyl Cellulose, Polyvinyl Alcohol, Calcium Alginate

Posted Date: February 12th, 2021

DOI: https://doi.org/10.21203/rs.3.rs-217915/v1

License: (9) This work is licensed under a Creative Commons Attribution 4.0 International License.

Read Full License 
1 A Novel Bilayer Propionyl-L-carnitine Loaded Polyvinyl Alcohol/Calcium Alginate/Carboxymethyl Cellulose wound dressing for the treatment of diabetic wounds: an in vitro and in vivo study

3 Wenjun Jiang ${ }^{1,5, a}$, Zuoyao Huang ${ }^{2, a}$, Weixiang Min $^{3,5, *}$, Hao Zhang ${ }^{4,5, * *}$

41 Department of Pediatric Surgery, Sichuan Provincial People's Hospital,University of Electronic Science and

5 Technology of China; Chengdu shichuan 610072 China

62 Department of Orthopaedics, Jinniu District People's Hospital of Chengdu; Chengdu Sichuan 610036, China

73 Department of anesthesiology, Sichuan Provincial People's Hospital, University of Electronic Science and

8 Technology of China; Chengdu shichuan 610072,China

9 4Department of Hepatobiliary Surgery, Sichuan Provincial People's Hospital, University of Electronic Science and

10 Technology of China; Chengdu shichuan 610072, China

115 Chinese Academy of Sciences Sichuan Translational Medicine Research Hospital, Chengdu 610072, China

$12 *$ Corresponding Author: Weixiang Min, E-mail:mmwwxx2020@163.com

$13 * *$ co-corresponding author Hao Zhang, E-mail:hawerchina@gmail.com

14

15 a Wenjun Jiang and Zuoyao Huang contributed equally to this work.

16

17

18

19

20

21

22

23

24

25

26

27 


\section{Abstract}

29 In the current study a drug delivering bilayer porous/nanofibrous wound dressing was developed using a combination

30 of electrospinning and freeze-drying methods. The wound dressings were prepared by lyophilization of 1:1 weight

31 ratios of calcium alginate and carboxymethyl cellulose (CMC) solutions. Drug delivering nanofibrous sheets were

32 fabricated by electrospinning of polyvinyl alcohol (PVA) solution incorporated with 1\%,3\%,5\%, and $10 \%$ of

33 Propionyl-L-carnitine. The dressings were studied regarding their microstructure, swelling capacity, mechanical

34 strength, surface wettability, water vapor permeability, drug release profile, in vitro degradation, cell viability assay,

35 hemocompatibility, porosity measurement, microbial penetration assay, and protein adsorption assay. Based on in

36 vitro studies, PVA sheets loaded with 5\% Propionyl-L-carnitine was chosen for the preparation of wound dressings.

37 The healing potential of the produced constructs was studied in rat model of diabetic wound. Our results showed that

38 the drug delivering dressings demonstrated significantly higher wound closure and better histological regeneration

39 compared to drug free constructs and sterile gauze. Our results suggest potential applicability of Propionyl-L-carnitine

40 delivering Calcium Alginate/CMC/PVA dressing for the treatment of diabetic wounds in clinic.

41 Key words: Diabetic wounds; Wound dressing; Propionyl-L-carnitine; Carboxymethyl Cellulose; Polyvinyl Alcohol;

42 Calcium Alginate

43

44

45

46

47

48

49

50

51

52

53

54

55

56

57 


\section{Declarations}

\section{$59 \quad$ Funding}

60 The authors did not receive support from any organization for the submitted work.

\section{Conflicts of interest}

62 The authors have no conflicts of interest to declare that are relevant to the content of this article.

\section{Ethics approval}

64 The animal studies were approved by ethics committee of University of Electronic Science and Technology of China 65 and were performed in accordance with the university guidelines.

66

67

68

69

70

71

72

73

74

75

76

77

78

79

80

81

82 


\section{Introduction}

85 Wounds are defined by integrity loss of skin tissue which can be caused by thermal, physical, or chemical injuries (Tarassoli et al. 2018). Given the fact that skin tissue has an inherent healing potential following injuries; in most cases, this tissue can repair its damages ( $Y u$ et al. 2019). However, in case of critical-sized defects or in disordered health conditions such as diabetes mellitus, wounds may turn into chronic non-healing wounds (Sahana and Rekha 2018). In diabetic patients, the angiogenesis and re-epithelialization processes are disturbed which hampers normal tissue repair responses. In addition, elevated inflammatory responses, poor blood flow, and oxidative damages result in a non-permissive environment for normal wound healing (Cho et al. 2019; Vijayakumar et al. 2019) . In such patients, the application of a proper wound care product seems crucial. Among various marketed products, the use of biopolymer-based wound dressings has gained significant attention during the past decades (Shah et al. 2019). In this regard, alginate has been widely exploited in various forms such as hydrogel, films, nanofibrous yarns, and composites to produce wound care material (Akbar et al. 2018; Jeong et al. 2020). Therapeutic appeal towards this polymer stems from its biocompatibility, biodegradability, non-immunogenicity, its high capacity to absorb wound exudates, and low cost (Aderibigbe and Buyana 2018). To further enhance the alginate's capacity to heal wounds; in the current research, Carboxymethyl cellulose (CMC) was blended with alginate to produce wound dressings. CMC can maintain a moist environment for wound healing and can absorb large amounts of wound exudates which is particularly favorable in treating diabetic wounds ( $\mathrm{Li}$ et al. 2016; Trevisol et al. 2019). Despite showing promising results in previous studies, the proposed wound dressing lacks enough bioactivity for a successful wound healing. Therefore, in this study we hypothesized using an electrospun Propionyl-L-carnitine-loaded Polyvinyl Alcohol (PVA) nanofibrous sheet as a drug delivery system to develop the wound care material. This drug is required for fatty acids metabolism in mitochondria. In addition, it has been shown that Propionyl-L-carnitine is an anti-oxidant agent which can protect cells from oxidative damages and can increase blood flow in ischemic tissues (Scioli et al. 2015). PVA is a hydrophilic biomaterial which its unique properties have made it an ideal career for hydrophilic drugs (Li et al. 2013; Orienti et al. 2001). Elctrospinng is a versatile and affordable method for scaffold fabrication. The prepared constructs have nanofibrous structures which have a strong resemblance to Extracellular Matrix architecture. Furthermore, the produced nanofibers have a relatively large surface area and can be exploited as a programmable drug delivery platform (Sill and Von Recum 2008). The aim of the current study is to evaluate the healing potential of a bilayer Calcium alginate/CMC/Propionyl-L-carnitine loaded PVA scaffolds in a rat model of diabetic wound.

\section{Methods and materials}

\section{Chemicals}

115 The materials and solvents were purchased from Sigma-Aldrich (St. Louis, USA) and Merck (Darmstadt, Germany), 116 respectively, unless otherwise noted.

\section{Fabrication of Calcium alginate/CMC films}


Firstly, sodium alginate of medium-viscosity (61\% of mannuronic and 39\% of guluronic acid) and CMC of medium viscosity (degree of substitution 0.7 ) were dissolved separately in glycerol containing distilled water (glycerol: polymer weight ratio was $60 \%$ ) at final concentration of $1.5 \%$ (wt $\%)$ and stirred for 24 hours at room temperature. Then, Calcium chloride at final concentration $2 \%(\mathrm{wt} \%)$ in distilled water was added to the alginate solution at volume ration of 1:4 to begin the cross linking process and stirred for further $24 \mathrm{~h}$ at room temperature. The equal amounts of each solution were blended to obtain a 1;1 alginate/CMC solution and stirred for $24 \mathrm{~h}$. The prepared mixture was transferred into $-20^{\circ} \mathrm{C}$ and incubated for $24 \mathrm{~h}$. The solidified sample was then kept at $-80{ }^{\circ} \mathrm{C}$ for further $24 \mathrm{~h}$. Then, the samples were freeze-dried (Telstar, Terrassa, Spain) for $48 \mathrm{~h}$.

\section{Fabrication of Propionyl-L-carnitine loaded PVA scaffolds}

127 Firstly, PVA $(\mathrm{Mw}=72 \mathrm{kDa})$ at final concentration of $12 \%$ was dissolved in distilled water for $24 \mathrm{~h}$ at room 128 temperature. Then, Propionyl-L-carnitine (at weight ratios of 1\%, 3\%,5\%, and 10\% was added to the PVA solution and thoroughly mixed for $8 \mathrm{~h}$. The prepared solution was transferred to a $10 \mathrm{ml}$ syringe connecting to an 18-gauge metal needle. The samples then fixed in the peristaltic pump of electrospinning device (Santa Marta co ltd, USA). The electrospinning began by applying a positive high voltage of 16 to $18 \mathrm{kv}$. The needle to mandrel distance set at $15 \mathrm{~cm}$ and the polymer feeding rate was $1 \mathrm{ml} / \mathrm{h}$. The electrospinning continued until the mandrel was fully covered by the nanofibers. The prepared sheets were cross-linked according to a method described previously (Stone et al. 2013).

\section{Scanning electron microscopy imaging of the samples}

To analyze the microstructure of the constructs, they were imaged under and SEM device (AIS2100, Seron sputter coater (SC7620, Quorum Technologies, England).

Swelling studies on Calcium alginate/CMC films

The swelling properties of the Calcium alginate/CMC films was studied using a method as described previously (Ehterami et al. 2019). Briefly, the freeze-dried samples were immersed in $10 \mathrm{ml}$ of phosphate buffer solution (PBS) and incubated for 3 days. After predetermined time points, the samples were taken out and immediately weighed. The swelling ratio of the samples was calculated according to the following equation.

143 Swelling ratio: $\frac{m 1-m 0}{m 0} \times 100$

144 Where $\mathrm{m} 1$ is the swollen weight of samples and $\mathrm{m} 0$ is the dry weight of the films.

\section{Mechanical strength measurement}

146 The mechanical properties of the scaffolds were studied using a uniaxial tensile testing device (Softon Technologies,

147 USA) at an extension rate of $1 \mathrm{~mm} / \mathrm{min}$,

\section{Contact angle measurement}


The surface hydrophilicity of the samples was studied using a static contact angle measuring device (KRUSS, Hamburg, Germany). A water droplet was placed on different spots of each scaffold and the angle between its surface and the scaffolds was calculated and averaged.

\section{Water vapor permeability study}

153 To assess the dressing's capability for gas transfer, this experiment was performed on scaffolds. $10 \mathrm{ml}$ of distilled

154 water was poured into an empty bottle and then capped by the fabricated dressings and then incubated at $33^{\circ} \mathrm{C}$ for 12 155 hours, the evaporated water through the scaffolds was calculated using the following equation.

156 Water vapor permeability $=\frac{W}{A T}$

157 Where w is mass of evaporated water, $\mathrm{A}$ is surface area, and $\mathrm{T}$ is the time of incubation.

158 In vitro degradation profile of the samples

159 The degradation rate of the scaffolds was studied by immersing predetermined amounts of each scaffold in $10 \mathrm{ml}$ of 160 PBS solution at $37^{\circ} \mathrm{C}$ under gentle shaking over a period of 10 days. After each time point, the samples were taken 161 out, dried, and weighed. Weight loss was measured according to the following equation.

162 Weight loss $=\frac{w 0-w 1}{w 0} \times 100$

163 where $\mathrm{W} 0$ is the initial weight of the films and W1 is the dry weight

164 after removal from the PBS solution.

Porosity assessment

166 The porosity of the scaffolds was studied using liquid displacement method. The samples were immersed in known 167 volume of ethanol and incubated for 1 hour. After this time period, samples were taken out and the residual ethanol 168 volume was recorded. The porosity of the scaffolds was calculated using the following equation.

169 Porosity $(\%)=\frac{v 1-v 3}{v 2-v 3} \times 100$

170 Where $\mathrm{v} 1$ is the initial volume of ethanol, v2 is the volume of ethanol after scaffolds immersion, and $\mathrm{v} 3$ is the volume 171 of ethanol after scaffold's removal.

\section{Release profile of Propionyl-L-carnitine from PVA films}

173 To evaluate the release profile of Propionyl-L-carnitine from PVA films, high-performance liquid chromatography 174 method was exploited as described previously (Marzo et al. 1988). Briefly, the drug loaded PVA fibers were immersed 175 in PBS solution for $24 \mathrm{~h}$ at $37^{\circ} \mathrm{C}$ under gentle shaking. At different time intervals, $1 \mathrm{ml}$ of the samples was obtained 176 and replaced with fresh PBS solution. The harvested medium was used to measure cumulative release profile of 177 Propionyl-L-carnitine from PVA fibers. 


\section{Microbial penetration assay}

181 In each group, $10 \mathrm{ml}$ bottles filled with $5 \mathrm{ml}$ of BHI broth culture medium was covered by the prepared constructs and incubated at room temperature. The invasion of the bacteria into the growth medium was studied at 3 and 7 days' time points. Vials capped with the sterile gauze and open vials were used as negative and positive controls, respectively. The blurred growth mediums as the indication of bacterial growth was analyzed using a spectroscopy method at 600 nm using a microplate spectrophotometer.

\section{Protein adsorption assay}

187 To evaluate the ability of the constructs to adsorb protein they were studied via batch contact method as described previously (Golafshan et al. 2017). Briefly, films with known weigh were immersed in PBS for $4 \mathrm{~h}$ and then weighed. Then, the swollen samples were immersed in $20 \mathrm{ml}$ of $0.2 \mathrm{wt} \%$ of Bovine Serum Albumin (BSA) solution and incubated at $37^{\circ} \mathrm{C}$ for 30 minutes under gentle shaking. The samples were taken out and the residual proteins in the supernatant was determined by spectrophotometric method at $280 \mathrm{~nm}$. The amount of adsorbed protein on the film's

192 surface was calculated using the following equation:

193 Adsorbed protein $(\mathrm{mg})=\frac{c 1-c 0}{w} \times \mathrm{V}$

194 Where $\mathrm{c} 1$ is the initial protein concentration of BSA, C0 is the protein concentration after scaffolds soaking and removal, $w$ is the weight of swollen scaffold, and V is the volume of BSA.

\section{Blood compatibility assay.}

$1978 \mathrm{ml}$ of whole blood was obtained from a healthy volunteer and mixed with $1 \mathrm{ml}$ of $3.8 \%$ Sodium citrate anticoagulant. 198 This sample was diluted with $2.5 \mathrm{ml}$ normal saline solution. $200 \mu \mathrm{l}$ of this blood was poured onto the samples and 199 incubated at $37{ }^{\circ} \mathrm{C}$ for $60 \mathrm{~min}$. Then, the scaffolds were taken out and the residual blood was centrifuged at $1500 \mathrm{rpm}$ 200 for $10 \mathrm{~min}$. The absorbance value of the samples was read at $545 \mathrm{~nm}$ using a Multi-Mode Microplate Reader. The 201 blood samples diluted with normal saline and distilled water was used as positive control and negative control respectively. The percentage of hemolysis was calculated using the following equation.

203 Percentage of hemolysis (\%): $\frac{D t-D n c}{D p c-D n c} \times 100$

204 Where Dt is the absorbance values of the studied group, Dnc and Dpc is the absorbance for negative control and 205 positive control respectively.

207 The MTT (3-(4, 5-dimethylthiazol-2-yl)-2, 5 diphenyl tetrazolium bromide, GIBCO-BRL, Eggenstein, Germany) test was exploited to study the proliferation rate of L-929 cells cultured on the constructs. Cells were seeded on drug 
loaded PVA sheets and Calcium alginate/CMC films at a density of $1 \times 10^{4}$ cells per sample and cultured for 7 days in Dulbecco's modified Eagle's medium: nutrient mixture F-12 supplemented with 10\% (v/v) fetal bovine serum, 100 unit $/ \mathrm{ml}$ of penicillin (Sigma-Aldrich, USA) and $100 \mu \mathrm{g} / \mathrm{ml}$ of streptomycin (Sigma-Aldrich, USA) in a humidified incubator at $37{ }^{\circ} \mathrm{C}$ with $5 \% \mathrm{CO}$. At each time point, the media on the samples was removed and replaced with 200 $\mu 1$ of $.5 \mathrm{mg} / \mathrm{ml}$ MTT solution and incubated for $4 \mathrm{~h}$ at a dark condition. After this time period, the MTT solution was discarded and replaced with $100 \mu \mathrm{DMSO}$ solution to dissolve any formed formazan crystals. The absorption values were recorded at $570 \mathrm{~nm}$.

\section{Animal studies}

The animal studies were approved by ethics committee of University of Electronic Science and Technology of China and were performed in accordance with the university guidelines. Diabetes mellitus was induced in adult male Wistar

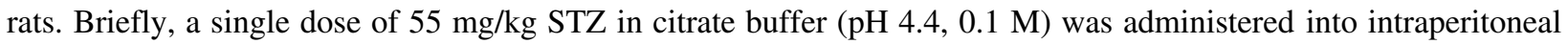
soft tissue and rats administered with citrate buffer served as control group. Blood samples were obtained from retroorbital plexus veins and the blood glucose level was measured. The rats whose serum glucose level was more than $250 \mathrm{mg} / \mathrm{dl}$ met the criteria for diabetes mellitus otherwise they were excluded from the study. For the creation of in vivo wound healing model, the rats were anesthetized by intraperitoneal injection of Ketamine 5\%/Xylazine 2\% (70 $\mathrm{mg}$ ketamine and $6 \mathrm{mg}$ Xylazine/1 kg body weight). The rats' back was shaved and disinfected using povidone iodine and a $1.5 * 1.5 \mathrm{~cm}^{2}$ of their skin was excised (Fig. 1). The rats were divided into 3 groups (5 animals per group) namely group A in which wounds were covered with Calcium alginate/CMC/ Propionyl-L-carnitine-loaded PVA scaffolds, group B in which the rats were treated with Calcium alginate/CMC/ Drug free PVA scaffolds, and negative control in which sterile gauze was used to cover the wounds. For wound dressing's application, PVA films were placed directly on the wound tissue then Calcium alginate/CMC films covered the PVA sheets. The whole bilayer wound dressing was fixed in place by using an elastic adhesive bandage. The wound dressings were changed on daily basis. To evaluate the wound size reduction, the macroscopic appearance of the wounds was imaged at day 7 and 14 using a digital camera (Canon Inc., Tokyo, Japan). The percentage of wound contraction was calculated using the following equation:

Percentage of wound closure $=\left(1-\frac{\text { open wound area }}{\text { initial } \text { wound area }}\right) \times 100$

After 14 days of post-wounding, the animals were humanely killed by ketamine overdose and the wound tissues were harvested for histopathological examinations. After processing and embedding in paraffin the samples were sectioned and underwent tissue staining with hematoxylin-eosin (H\&E) and Masson's trichrome (MT). To prevent bias, an independent pathologist reported the pathological changes under a light microscope (Carl Zeiss, Thornwood, USA) with a digital camera (Olympus, Tokyo, Japan). Epithelial tissue thickness, recruitment of macrophages, fibroplasia, 
and new blood vessel formation were studied. Furthermore, collagen deposition was also measured according to histopathological images.

\section{Results}

\section{Microstructure studies}

247 The microstructure of the scaffolds was observed using scanning electron microscopy imaging. The results (Fig. 2 a) showed the Calcium alginate/CMC films had porous structure with interconnectivity of the pores. The pore size measurement using Image J software (National Institutes of Health, Bethesda, USA) showed that the average pore size was around $50-100 \mu \mathrm{m}$. This porous structure of the membranes can facilitate gas exchanges and prevent wound maceration. This would inhibit establishing a non-aerobic condition for bacterial growth. In addition, this porous structure would also facilitate wound exudates absorption (Karahaliloglu et al. 2017; Komatsuzaki et al. 1994). Fig. 2 b shows microstructure for PVA scaffolds loaded with 5\% Propionyl-L-carnitine. As shown, the nanofibers had randomly oriented structure with smooth morphology. Twenty points of images were selected for fiber size measurement and the results showed that the fibers had $1136.03 \pm 52.67 \mathrm{~nm}$ diameter. Structural resemblance of the produced scaffolds to native extracellular matrix architecture is particularly favorable for stimulating fibroblasts' migration, attachment, and proteins production which will have a profound effect in wound closure (Chen et al. 2008;

258 Gu et al. 2009).

\section{Swelling studies of Calcium alginate/CMC films}

260 Swelling study of the membranes was conducted to test their ability to form hydrogel and marinating a moist 261 environment upon exudates absorption. Non-cross linked alginate polymer will turn into sol when it absorbs water 262 while when cross linked with calcium ion it will be able to form hydrogel upon water absorption (Novikova et al. 263 2006). Fig. 3 illustrates time course swelling of Calcium alginate/CMC membranes in $72 \mathrm{~h}$ time period. As shown, 264 the percentage of swelling reaches to the highest level of $362.33 \pm 15.29 \%$ after $4 \mathrm{~h}$ of scaffold immersion and then 265 decreases to the $111.66 \pm 8.97 \%$ at $72 \mathrm{~h}$. As the dressings are changed on daily basis therefore they can absorb the 266 wound exudates to their utmost capacity within few hours of dressing's application.

\section{Cumulative release profile of Propionyl-L-carnitine from PVA nanofibers}

268 The ability of the bioactive dressing to sustain release the loaded drug in the wound bed is of paramount importance. As shown in Fig. 4, the cumulative release of Propionyl-L-carnitine from PVA films could reach to $80.06 \pm 4.38 \%$ after $24 \mathrm{~h}$ of incubation. Therefore, the designed delivery system will be able to deliver almost all the loaded drug to

271 the wound bed in a sustained manner. The drug release from polymeric matrices has a direct relation with the

272 degradation rate. Given the fact that PVA is a fast-degrading polymer; even after cross linking, this polymer is 273 particularly interesting in developing drug delivering wound dressings since they are usually replaced on daily basis 274 (Hamad et al. 2014). 


\section{Degradation rate measurement}

276 The in vitro degradation study showed that the percentage of weight loss for Calcium alginate/CMC scaffolds could

277 reach to $44.39 \pm 3.37 \%$ and $55.72 \pm 69 \%$ after 5 days and 10 days respectively. While, PVA sheets almost fully

278 degraded within $24 \mathrm{~h}$ of incubation in PBS solution.

Porosity measurement

Porosity evaluation showed that the Calcium alginate/CMC scaffolds had $83.57 \pm 4.38 \%$ which is ideal for a tissue engineered construct (Salehi et al. 2018).

\section{Blood compatibility test}

283 Results of hemolysis assay of different samples are shown in Fig. 5. The study revealed that the Calcium alginate/CMC

284 membrane had significantly higher OD values compared to PVA sheets and negative control groups. This could be 285 due to hemostatic effects of calcium alginate which is in accordance with previous studies. Calcium Alginate can 286 promote blood coagulation and cause hemostasis which is the first phase of wound healing (TAŞKIN et al. 2013). 287 Positive control had significantly higher absorbance values compared to other groups.

\section{Cell viability assay}

289 To study the metabolic activity and proliferation rate of L929 cells on different scaffolds, MTT assay was conducted 290 at days 1, 3, and 7. As shown in Fig. 6 a, there was no significant deference between Calcium alginate/CMC films and 291 control group at different time intervals, implying that the prepared construct was not toxic against L929 cells. In 292 drug-loaded PVA groups (Fig. 6 b), at day 1 PVA fibers incorporated with 5\% Propionyl-L-carnitine had significantly 293 higher absorbance values compared to other groups. This trend was almost unchanged towards the day 7. According 294 to this assay, we assumed that 5\% of Propionyl-L-carnitine was optimal concentration which had a beneficial effect 295 on fibroblasts' metabolic activity. Therefore, this concentration was chosen for treating skin wounds.

\section{Microbial penetration assay}

298 An ideal wound care product should prevent microbial invasion into the wound bed (Samadian et al. 2020). Our results 299 showed that (Fig. 7) the bilayer wound dressing could significantly reduce bacterial colony formation in the growth 300 medium. This can be due to physical barrier that PVA nanofibers may have caused. In addition, Wong et al. used 301 CMC films for controlling bacterial wound infection. Infected wounds were treated with CMC films and it was 302 revealed that the membranes could attach to the bacteria and remove them from the wound tissue (Wong and Ramli

303 2014). Therefore, it is hypothesized that the CMC polymer in the bilayer wound dressing attached to the bacteria and 304 prevented them from invading the growth medium.

305 Water vapor permeability study 
Interestingly; in our fabrication method, the water vapor transmission capability of the dressings can be tailed to obtain the optimal moist environment for wound healing. This can be achieved through tailoring the polymer concentrations, altering the freeze-drying parameters, or using filler materials. Our study showed that the bilayer wound dressing had $11.98 \pm 1.37 \mathrm{mg} / \mathrm{cm}^{2} / \mathrm{h}$ of water vapor permeation.

\section{Contact angle measurement}

Generally, hydrophobic materials are not suitable for wound dressing applications since they lack enough capacity for water absorption (Jin et al. 2016). Therefore, surface contact angle measurement was performed to evaluate the dressings surface wettability. The results showed that the PVA/5\% Propionyl-L-carnitine had $64.39 \pm 4.28^{\circ}$ and Calcium alginate/CMC films had $56.19 \pm 2.15^{\circ}$ of contact angle.

\section{Tensile strength measurement}

An acceptable mechanical strength is essential for wound dressing handling during the dressing's application. Our study revealed that the prepared constructs' mechanical properties were in acceptable range which is from 0.7 to 18 Mpa (Samadian et al. 2020). The PVA/5\% Propionyl-L-carnitine sheets had $2.68 \pm .24$ Mpa tensile strength and Calcium alginate/CMC films had $3.53 \pm .67 \mathrm{Mpa}$ tensile strength.

\section{Protein adsorption assay}

At the initial phases of thrombosis on a biomaterial surface, proteins are adsorbed and initiate a series of events which eventually cause thrombosis. Therefore, better protein adsorption properties are pro-thrombotic for a wound dressing (Golafshan et al. 2017). Our data showed that the amount of adsorbed protein to PVA/5\% Propionyl-L-carnitine sheets was around $3.25 \pm .49 \mathrm{mg}$ while the Calcium alginate/CMC films could adsorb $5.84 \pm .35 \mathrm{mg}$ to its surface. When a biomaterial is exposed to biological fluids its surface gets hydrated and a thin layer is formed which leads to adsorption of series of different proteins. This phenomenon depends on the surface chemistry and the higher the hydrophilicity, the lower the protein adsorption. Since by increasing the surface wettability the energetic cost for surface dehydration also increases hence lowering the protein replacement at the surface (Vogler 2012).

\section{In vivo wound healing}

The healing potential of the bilayer wound dressing was investigated in a rat model od diabetic wound. Fig. 8 a shows the percentage of wound closure for wounds treated with different materials. As shown, the rats treated with drug loaded wound dressing had significantly higher wound closure compared to drug free composites and negative control groups. The percentage of wound contraction for drug loaded wound dressing could reach to $67.39 \pm 5.26 \%$ and $92.49 \pm 6.19 \%$ at 7 days and 14 days post-wounding respectively. While, this value for drug free membranes was $56.49 \pm 3.38 \%$ and $79.67 \pm 4.29 \%$ at day 7 and 14 of surgery respectively. Histopathological examinations evaluated the healing potential of different groups and the results are shown in Figure 9. In the negative control group, the presence of inflammatory cells and disintegrated collagen depositions was evident. In addition, epidermal layer is not formed in this group. In Calcium alginate/CMC/PVA 5\% Propionyl-L-carnitine group, epithelialization had been completed and less inflammatory cells were observed. Moreover, skin appendices regeneration was observed in this 
group as evidenced by the presence of normal rete ridges, sebaceous glands, and hair follicles. Overall structure of the repaired skin in this group had more resemblance to positive control group. The histomorphometric study revealed that the epithelial thickness (Fig. 8 b) in drug delivering wound dressing was significantly higher compared to other groups. The epithelial thickness for Calcium alginate/CMC/PVA 5\% Propionyl-L-carnitine group was $48.13 \pm 5.49$ $\mu \mathrm{m}$. While, Calcium alginate/CMC/PVA and negative control groups exhibited the epithelial thickness of $31.52 \pm$ $6.87 \mu \mathrm{m}$ and $6.19 \pm 1.61 \mu \mathrm{m}$ respectively. Wound tissue in the negative control group had immature granulation tissue which was evidenced by ineffective wound closure. The wound contraction heavily depends on deposition of collagen molecules. Study revealed that the percentage of collagen deposition (Fig. 8 c) in Calcium alginate/CMC/PVA 5\% Propionyl-L-carnitine group was significantly greater than Calcium alginate/CMC/PVA group $(78.31 \pm 7.26 \%$ vs. $64.59 \pm 6.01 \%$, p value $<0.05)$.

\section{Discussion}

The main goal of skin tissue engineering is to develop products which results in prompt aesthetic and functional recovery of skin with negligible scarring (Mansbridge 2008). In this way, tissue engineered wound dressings need to have versatile properties to successfully support tissue repair and manage different phases of wound healing (Chen et al. 2009). Wound healing is a complex process which is composed of different interrelated phases known as hemostasis, inflammatory phase, proliferation phase, and remodeling phase (George Broughton et al. 2006). In every step of wound healing, specific biomaterials and signaling molecules can be used to drive the healing process. In this regard, a variety of multi-potential wound dressings have been tested in previous studies (Boateng et al. 2008). For designing such dressings, material selection and optimization is the key step. Among different types of biomaterials, we exploited combined use Calcium alginate, CMC, and PVA to fabricate a dug delivering bilayer membrane. Alginate is a natural biomaterial that is harvested from brown seaweed. Its low cost, biocompatibility and non-toxicity has added to its therapeutic appeal for wound management applications (Aderibigbe and Buyana 2018). Dressings produced from this polymer maintain a moist environment conductive to fibroblasts and keratinocytes proliferation, migration, and functions (Zhang and Zhao 2020). CMC is another polysaccharide which can be exploited to promote wound healing. it can absorb huge amounts of wound exudates and can impart antibacterial properties to the fabricated dressing. In addition, dressings produced from this polymer can promote angiogenesis and autolytic debridement (Kanikireddy et al. 2020). To facilitate fast release of Propionyl-L-carnitine into the wound bed we chose PVA for our drug delivery matrix. Our results showed that sheets produced from PVA could successfully release its drug cargo within $24 \mathrm{~h}$. For fabrication of the dressings, we used a combination of freeze-drying and electrospinning methods. Lyophilization results in a porous structure which facilitates gas exchange and exudate absorption (Gonzaga et al. 2020). While, the electrospinning method produce scaffolds with structural resemblance to native extracellular matrix. In addition, the fabricated fibers have a high surface to volume ratio which make them ideal carriers for a variety of drugs (Khalf and Madihally 2017). Although the proposed biomaterials have unique properties for wound healing, they lack enough bioactivity. To impart this property to the dressings, Propionyl-L-carnitine was incorporated into PVA nanofibers using electrospinning method. This substance is an ester of L-carnitine which is essential in fatty 
acids metabolism. Furthermore, it has been shown that Propionyl-L-carnitine is an antioxidant agent and can prevent lipid peroxidation in elevated levels of reactive oxygen species (Di Emidio et al. 2020).

The healing potential of Propionyl-L-carnitine loaded bilayer wound dressing was evaluated in rat model of diabetic wound. The results showed that the drug delivering dressing group had significantly higher wound size reduction, epithelial thickness, and collagen deposition. Diabetic wounds are often accompanied by endothelial dysfunction and impaired blood flow (Kolluru et al. 2012). Pola et al. showed that Propionyl-L-carnitine can dramatically reduce wound size in patients with venous leg ulcer which was accompanied by enhanced blood flow in the patients (Pola et al. 1991). In addition, Stasi et al. showed that Propionyl-L-carnitine can improve blood flow and vascular function in a rabbit model of hind limb ischemia. They showed that Propionyl-L-carnitine had increased vasodilation through increasing nitric oxide (NO) plasma levels hence causing vasodilation in ischemic sites and formation of new blood vessels (Stasi et al. 2010). Scioli et al. reported that Propionyl-L-carnitine oral administration could improve skin flap viability. They could show that upregulation of inducible nitric oxide synthase (iNOS), vascular endothelial growth factor (VEGF), placental growth factor (PIGF) and reduction of NADPH-oxidase 4 (Nox4) expression. Endothelial dysfunction which is the major cause of diabetic wounds can be prevented through Nox4 inhibition (Scioli et al. 2015). Therefore, we assume that part of the observed healing effect could be due to inhibiting endothelial dysfunction and increasing blood flow in the wound site. Elevated levels of Reactive Oxygen Species (ROS) are associated with delayed wound healing in diabetic patients (Nouvong et al. 2016). Previous studies suggest the application of radical scavengers to modulate ROS levels in treating diabetic wounds (Naseri-Nosar et al. 2017). Vanella et al. showed that Propionyl-L-carnitine has radical scavenger, anti-oxidant, and DNA protective effects (Vanella et al. 2000). Furthermore, Gomez-amorez proved anti-oxidant activities of Propionyl-L-carnitine in liver and heart of spontaneously hypertensive rats (Gómez-Amores et al. 2006). Therefore, the anti-oxidant potential of this drug may also have contributed in the observed healing effects. However, more extensive researches at gene and protein levels need to be performed to further elucidate the underlying mechanisms of Propionyl-L-carnitine's contribution in treating diabetic wounds.

\section{Conclusion}

In summary, a bilayer drug loaded porous/nanofibrous polymeric wound dressing was fabricated in this study for the management of diabetic wounds. Our results revealed that scaffolds loaded with 5\% Propionyl-L-carnitine exhibited the highest cell proliferation with fibroblasts. In vivo studies showed that the drug loaded wound dressing had significantly higher healing potential compared to drug free dressings and negative control. This preliminary study suggests potential applicability of this dressing to treat diabetic wounds in clinic.

\section{Conflicts of interest}

The authors declare no conflicts of interest with regard to this study. 


\section{References}

411 Aderibigbe BA, Buyana B (2018) Alginate in wound dressings Pharmaceutics 10:42

412

413

414

415

416

417

418

419

420

421

422

423

424

425

426

427

428

429

430

431

432

433

434

435

436

437

438

439

440

441

442

443

444

445

Akbar MU, Zia KM, Akash MSH, Nazir A, Zuber M, Ibrahim M (2018) In-vivo anti-diabetic and wound healing potential of chitosan/alginate/maltodextrin/pluronic-based mixed polymeric micelles: Curcumin therapeutic potential INT J BIOL MACROMOL 120:2418-2430

Boateng JS, Matthews KH, Stevens HN, Eccleston GM (2008) Wound healing dressings and drug delivery systems: a review J PHARM SCI 97:2892-2923

Chen J-P, Chang G-Y, Chen J-K (2008) Electrospun collagen/chitosan nanofibrous membrane as wound dressing COLLOID SURFACE A 313:183-188

Chen M, Przyborowski M, Berthiaume F (2009) Stem cells for skin tissue engineering and wound healing CRIT REV BIOMED ENG 37

Cho H, Blatchley MR, Duh EJ, Gerecht S (2019) Acellular and cellular approaches to improve diabetic wound healing ADV DRUG DELIVER REV 146:267-288

Di Emidio G et al. (2020) Regulatory Functions of L-Carnitine, Acetyl, and Propionyl L-Carnitine in a PCOS Mouse Model: Focus on Antioxidant/Antiglycative Molecular Pathways in the Ovarian Microenvironment Antioxidants 9:867

Ehterami A et al. (2019) Chitosan/alginate hydrogels containing Alpha-tocopherol for wound healing in rat model J DRUG DELIV SCI TEC 51:204-213

George Broughton I, Janis JE, Attinger CE (2006) Wound healing: an overview PLAST RECONSTR SURG 117:1e-S-32e-S

Golafshan N, Rezahasani R, Esfahani MT, Kharaziha M, Khorasani S (2017) Nanohybrid hydrogels of laponite: PVA-Alginate as a potential wound healing material CARBOHYD POLYM 176:392-401

Gómez-Amores L, Mate A, Revilla E, Santa-María C, Vázquez CM (2006) Antioxidant activity of propionylL-carnitine in liver and heart of spontaneously hypertensive rats LIFE SCI 78:1945-1952

Gonzaga VdA et al. (2020) Chitosan-laponite nanocomposite scaffolds for wound dressing application J BIOMED MATER RES B 108:1388-1397

Gu S-Y, Wang Z-M, Ren J, Zhang C-Y (2009) Electrospinning of gelatin and gelatin/poly (I-lactide) blend and its characteristics for wound dressing MAT SCI ENG C 29:1822-1828

Hamad D, Mehrvar M, Dhib R (2014) Experimental study of polyvinyl alcohol degradation in aqueous solution by UV/H2O2 process POLYM DEGRAD STABIL 103:75-82

Jeong S, Kim B, Park M, Ban E, Lee S-H, Kim A (2020) Improved Diabetic Wound Healing by EGF Encapsulation in Gelatin-Alginate Coacervates Pharmaceutics 12:334

Jin SG et al. (2016) Influence of hydrophilic polymers on functional properties and wound healing efficacy of hydrocolloid based wound dressings INT J PHARMACEUT 501:160-166

Kanikireddy V, Varaprasad K, Jayaramudu T, Karthikeyan C, Sadiku R (2020) Carboxymethyl cellulosebased materials for infection control and wound healing: A review INT J BIOL MACROMOL 
Karahaliloglu Z, Kilicay E, Denkbas EB (2017) Antibacterial chitosan/silk sericin 3D porous scaffolds as a wound dressing material ARTIF CELL NANOMED B 45:1172-1185

Khalf A, Madihally SV (2017) Recent advances in multiaxial electrospinning for drug delivery EUR J PHARM BIOPHARM 112:1-17

Kolluru GK, Bir SC, Kevil CG (2012) Endothelial dysfunction and diabetes: effects on angiogenesis, vascular remodeling, and wound healing INT VASC MED 2012

Komatsuzaki S, Hirayama T, Toyokawa T (1994) Wound dressing having a porous structure. Google Patents,

Li D, Ye Y, Li D, Li X, Mu C (2016) Biological properties of dialdehyde carboxymethyl cellulose crosslinked gelatin-PEG composite hydrogel fibers for wound dressings CARBOHYD POLYM 137:508-514

Li X, Kanjwal MA, Lin L, Chronakis IS (2013) Electrospun polyvinyl-alcohol nanofibers as oral fast-dissolving delivery system of caffeine and riboflavin COLLOID SURFACE B 103:182-188

Mansbridge J (2008) Skin tissue engineering J BIOMAT SCI-POLYM E 19:955-968

Marzo A, Monti N, Ripamonti M, Martelli EA (1988) Application of high-performance liquid chromatography to the analysis of propionyl-L-carnitine by a stereospecific enzyme assay $\mathrm{J}$ CHROMATOGR A 459:313-317

Naseri-Nosar M, Farzamfar S, Sahrapeyma H, Ghorbani S, Bastami F, Vaez A, Salehi M (2017) Cerium oxide nanoparticle-containing poly ( $\varepsilon$-caprolactone)/gelatin electrospun film as a potential wound dressing material: in vitro and in vivo evaluation MATER SCI ENG C 81:366-372

Nouvong A, Ambrus AM, Zhang ER, Hultman L, Coller HA (2016) Reactive oxygen species and bacterial biofilms in diabetic wound healing PHYSIOL GENOMICS

Novikova LN, Mosahebi A, Wiberg M, Terenghi G, Kellerth JO, Novikov LN (2006) Alginate hydrogel and matrigel as potential cell carriers for neurotransplantation J BIOMED MATER RES A 77:242-252

Orienti I, Trere R, Zecchi V (2001) Hydrogels formed by cross-linked polyvinylalcohol as colon-specific drug delivery systems DRUG DEV IND PHARM 27:877-884

Pola P, Flore R, Serricchio M, Tondi P (1991) New carnitine derivatives for the therapy of cutaneous ulcers in vasculopathics DRUG EXP CLIN RES 17:277-282

Sahana T, Rekha P (2018) Biopolymers: Applications in wound healing and skin tissue engineering MOL BIOL REP 45:2857-2867

Salehi M et al. (2018) Sciatic nerve regeneration by transplantation of Schwann cells via erythropoietin controlled-releasing polylactic acid/multiwalled carbon nanotubes/gelatin nanofibrils neural guidance conduit J BIOMED MATER RES B 106:1463-1476

Samadian $\mathrm{H}$ et al. (2020) Electrospun cellulose acetate/gelatin nanofibrous wound dressing containing berberine for diabetic foot ulcer healing: in vitro and in vivo studies SCI REP 10

Scioli MG, Giudice PL, Bielli A, Tarallo V, De Rosa A, De Falco S, Orlandi A (2015) Propionyl-L-carnitine enhances wound healing and counteracts microvascular endothelial cell dysfunction PLoS One 10:e0140697 
Shah SA et al. (2019) Biopolymer-based biomaterials for accelerated diabetic wound healing: A critical review INT J BIOL MACROMOL 139:975-993

Sill TJ, Von Recum HA (2008) Electrospinning: applications in drug delivery and tissue engineering Biomaterials 29:1989-2006

Stasi MA et al. (2010) Propionyl-I-carnitine improves postischemic blood flow recovery and arteriogenetic revascularization and reduces endothelial NADPH-oxidase 4-mediated superoxide production ARTERIOSCL THROM VAS 30:426-435

Stone SA, Gosavi P, Athauda TJ, Ozer RR (2013) In situ citric acid crosslinking of alginate/polyvinyl alcohol electrospun nanofibers MATER LETT 112:32-35

Tarassoli SP, Jessop ZM, Al-Sabah A, Gao N, Whitaker S, Doak S, Whitaker IS (2018) Skin tissue engineering using 3D bioprinting: an evolving research field J PLAST RECONSTR AES 71:615-623

TAŞKIN AK et al. (2013) The hemostatic effect of calcium alginate in experimental splenic injury model Ulus Travma Acil Cerrahi Derg 19:195-199

Trevisol T, Fritz A, de Souza S, Bierhalz A, Valle J (2019) Alginate and carboxymethyl cellulose in monolayer and bilayer films as wound dressings: Effect of the polymer ratio J APPL POLYM SCI 136:46941

Vanella A, Russo A, Acquaviva R, Campisi A, Di Giacomo C, Sorrenti V, Barcellona M (2000) L-propionylcarnitine as superoxide scavenger, antioxidant, and DNA cleavage protector CELL BIOL TOXICOL 16:99-104

Vijayakumar V, Samal SK, Mohanty S, Nayak SK (2019) Recent advancements in biopolymer and metal nanoparticle-based materials in diabetic wound healing management INT J BIOL MACROMOL 122:137-148

Vogler EA (2012) Protein adsorption in three dimensions Biomaterials 33:1201-1237

Wong TW, Ramli NA (2014) Carboxymethylcellulose film for bacterial wound infection control and healing CARBOHYD POLYM 112:367-375

Yu JR et al. (2019) Current and future perspectives on skin tissue engineering: Key features of biomedical research, translational assessment, and clinical application ADV HEALTHC MATER 8:1801471

Zhang M, Zhao X (2020) Alginate hydrogel dressings for advanced wound management INT J BIOL MACROMOL 


\section{$518 \quad$ Figure captions}

519 Fig. 1 Full thickness wound healing model in diabetic Wistar rats

520 Fig. 2 Scanning electron microscopy images of (a) Calcium alginate/CMC films, (b) PVA fibers lloaded with 5\% 521 Propionyl-L-carnitine

522 Fig. 3 The swelling percentages of Calcium alginate/CMC films over a period of $72 \mathrm{~h}$

523 Fig. 4 Cumulative release profile of Propionyl-L-carnitine from PVA nanofibers

524 Fig. 5 Histogram comparing the blood compatibility of the experimental samples. Values represent the mean \pm SD, $\mathrm{n}$ $525=4, * \mathrm{P}<0.05, * * \mathrm{P}<0.01$ and $* * * \mathrm{P}<0.005$

526 Fig. 6 Histogram comparing the viability of L929 cells on (a) Calcium alginate/CMC films and (b) on PVA fibers at

5271 day, 3 days, and 7 days after cell seeding. Values represent the mean $\pm \mathrm{SD}, \mathrm{n}=3, * \mathrm{P}<0.05, * * \mathrm{P}<0.01$ and

$528 * * * * \mathrm{P}<0.005$

529 Fig.7 Microbial barrier property of the bilayer wound dressing after 3 and 7 days of incubation measured by 530 Spectrophotometer at $600 \mathrm{~nm}$

531 Fig. 8 Histogram comparing (a) percentage of wound closure at 7 days and 14 days post-wounding of different groups,

532 (b) epithelial thickness of different experimental groups, and (c) the percentage of collagen deposition in different 533 groups. Values represent the mean $\pm \mathrm{SD}, \mathrm{n}=5, * \mathrm{P}<0.05$, **P $<0.01$ and $* * * \mathrm{P}<0.005$

534 Fig. 9 Hematoxylin and Eosin (H\&E) and Masson's trichrome (MT) stained microscopic sections (200x 535 magnification) of wound tissues treated with different dressings 14 days' post-treatment. Thick arrows: epidermal 536 thickness, thin arrows: recruitment of inflammatory cells, arrowheads: skin appendages, red arrows: 537 neovascularization 


\section{Figures}

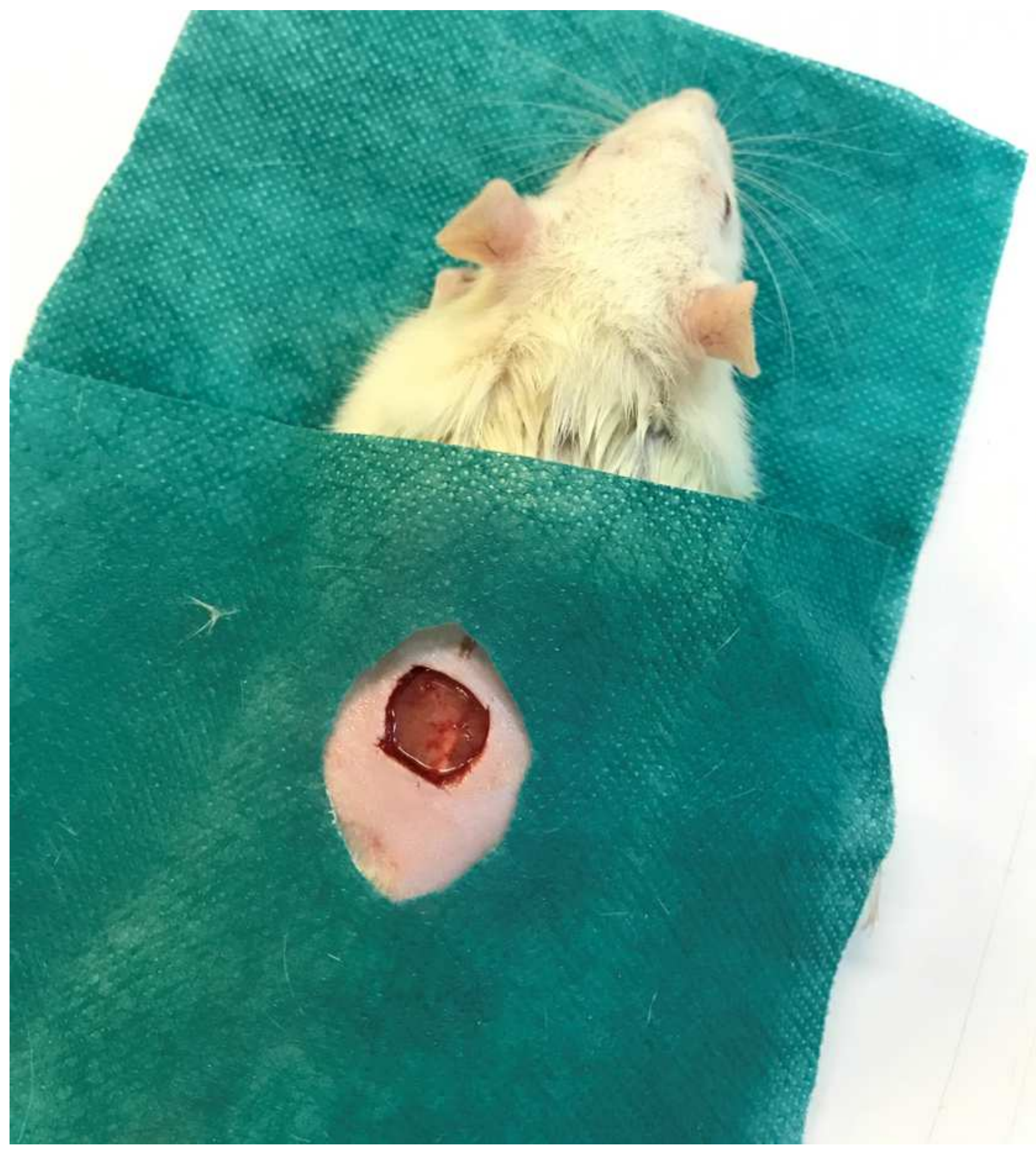

\section{Figure 1}

Full thickness wound healing model in diabetic Wistar rats 

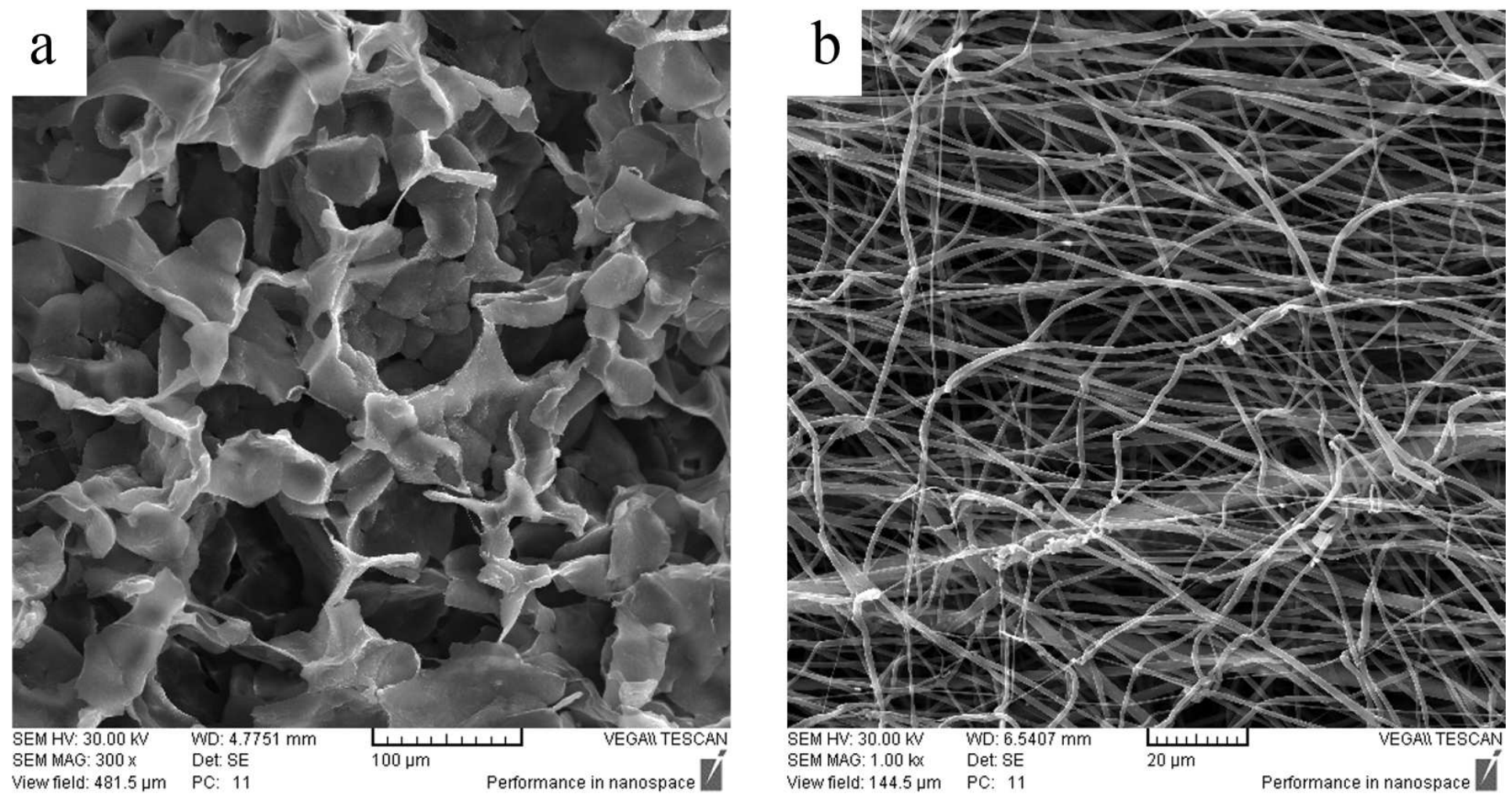

Figure 2

Scanning electron microscopy images of (a) Calcium alginate/CMC films, (b) PVA fibers lloaded with 5\% Propionyl-L-carnitine 


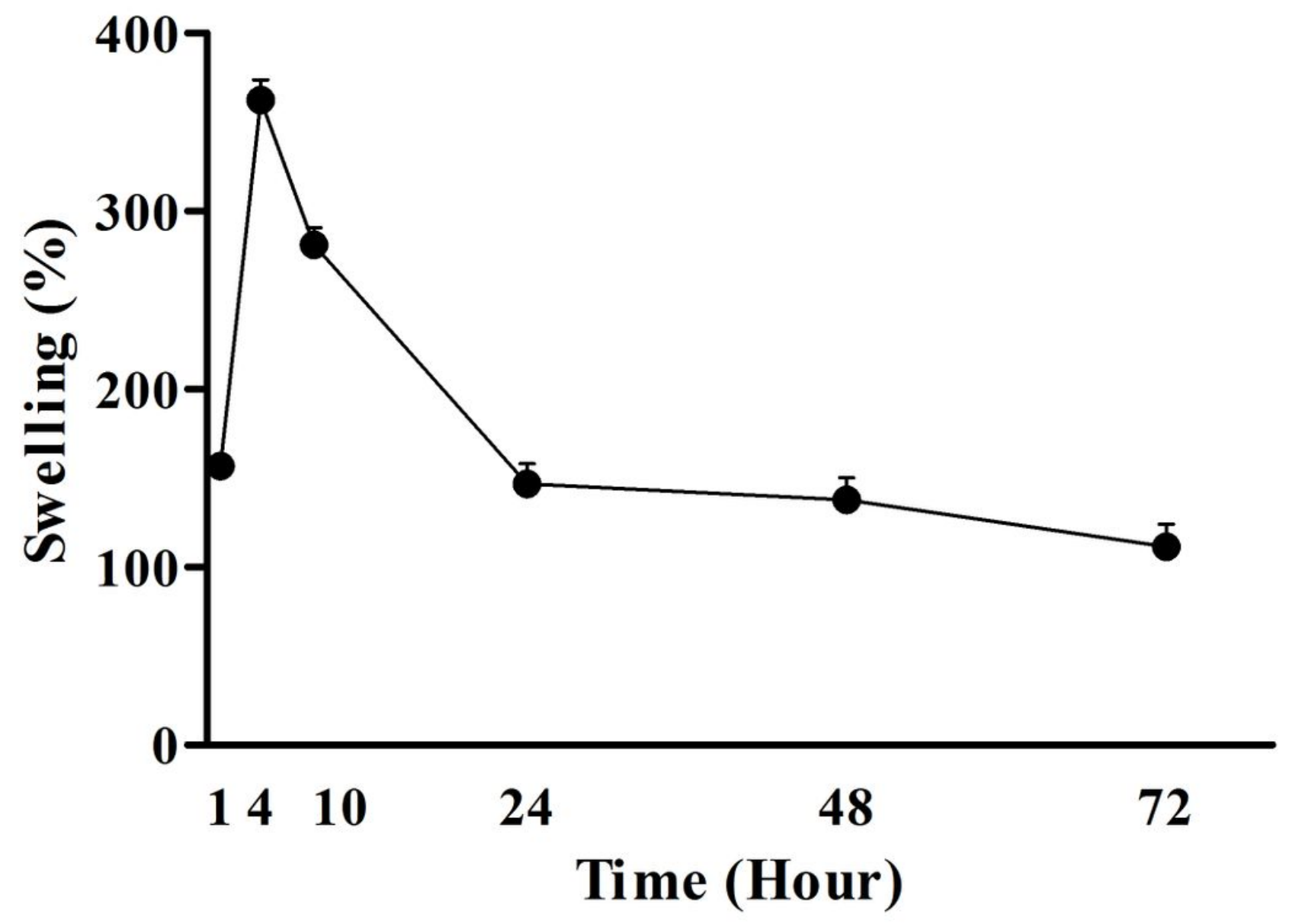

Figure 3

The swelling percentages of Calcium alginate/CMC films over a period of $72 \mathrm{~h}$ 


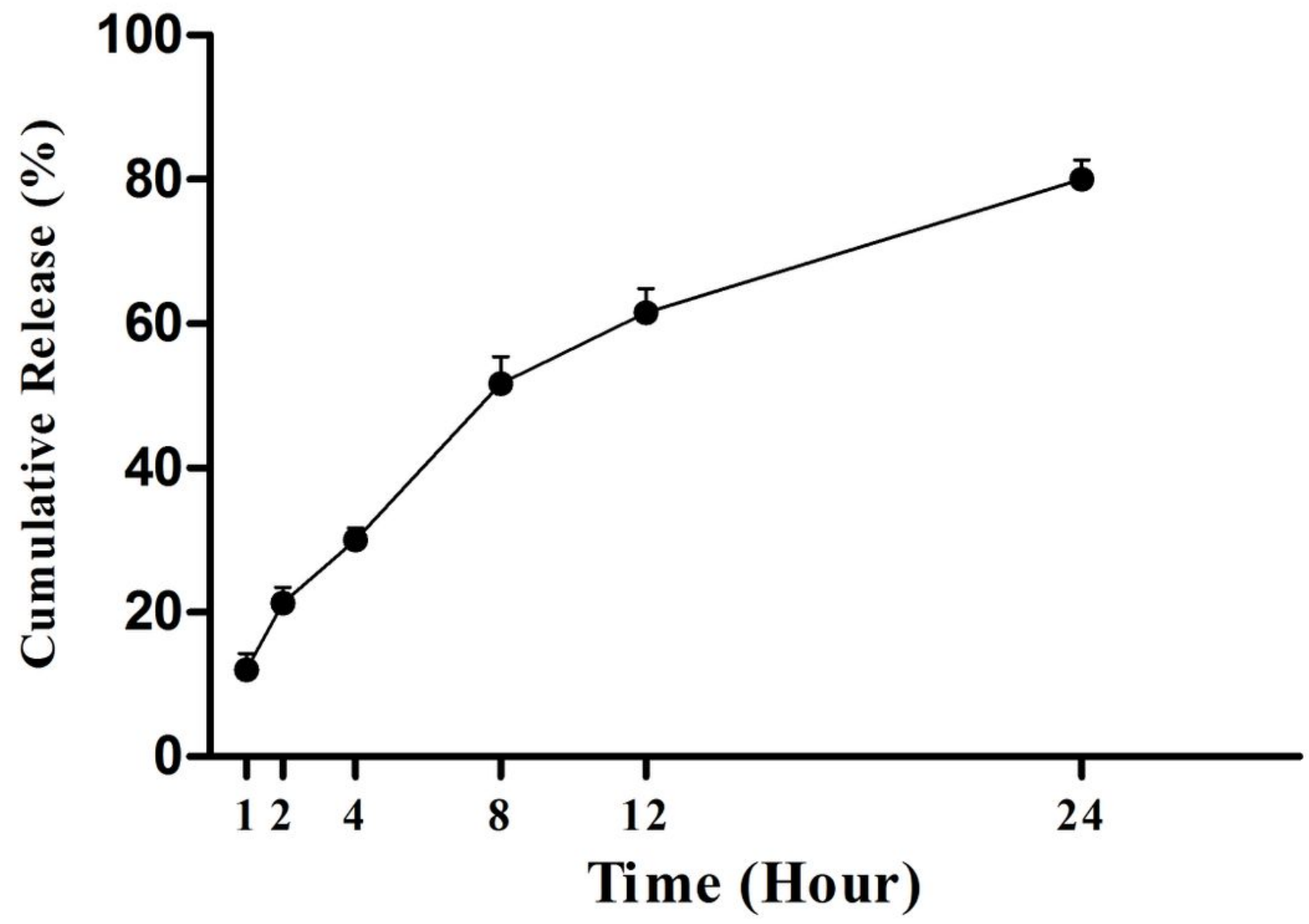

Figure 4

Cumulative release profile of Propionyl-L-carnitine from PVA nanofibers

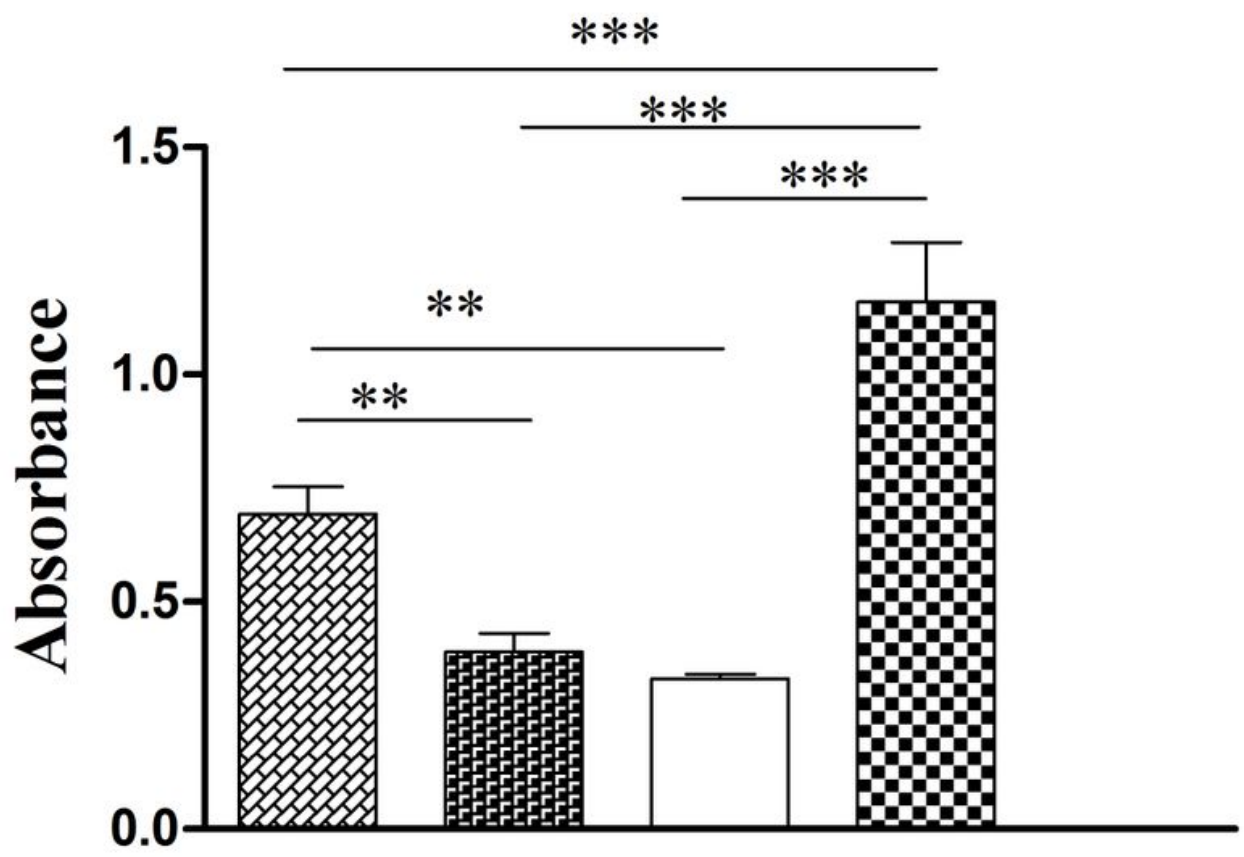

Eszy Alginate/CMC 茞秙 Drug-loaded PVA $\square$ Negative Control Q0. Positive Control 
Figure 5

Histogram comparing the blood compatibility of the experimental samples. Values represent the mean \pm $\mathrm{SD}, \mathrm{n}=4,{ }^{*} \mathrm{P}<0.05,{ }^{*} \mathrm{P}<0.01$ and ${ }^{*} * \mathrm{P}<0.005$
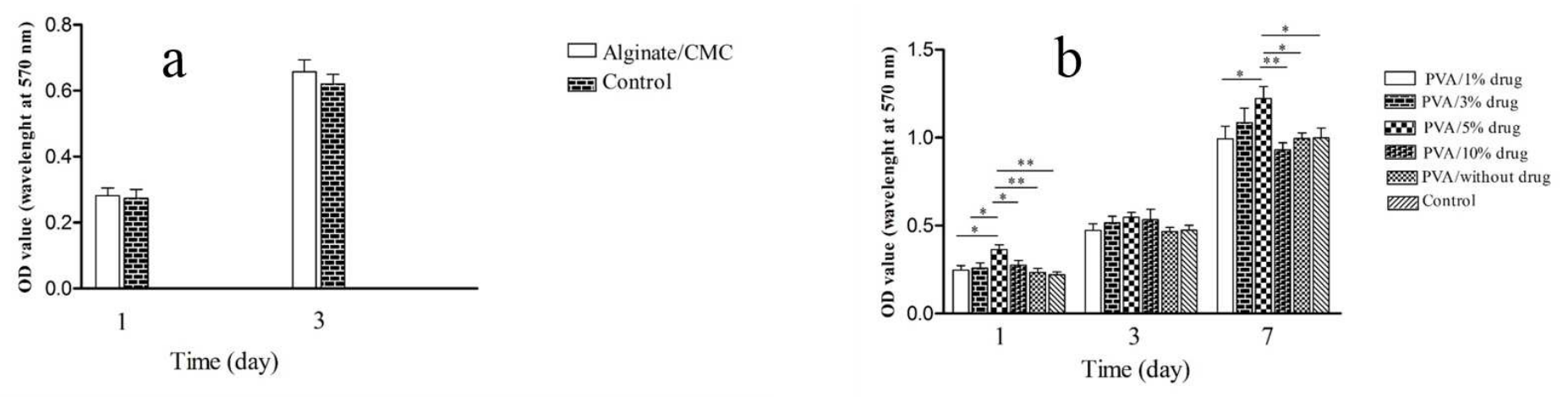

\section{Figure 6}

Histogram comparing the viability of L929 cells on (a) Calcium alginate/CMC films and (b) on PVA fibers at 1 day, 3 days, and 7 days after cell seeding. Values represent the mean $\pm S D, n=3, * P<0.05, * * P<0.01$ and ${ }^{* \star *} \mathrm{P}<0.005$

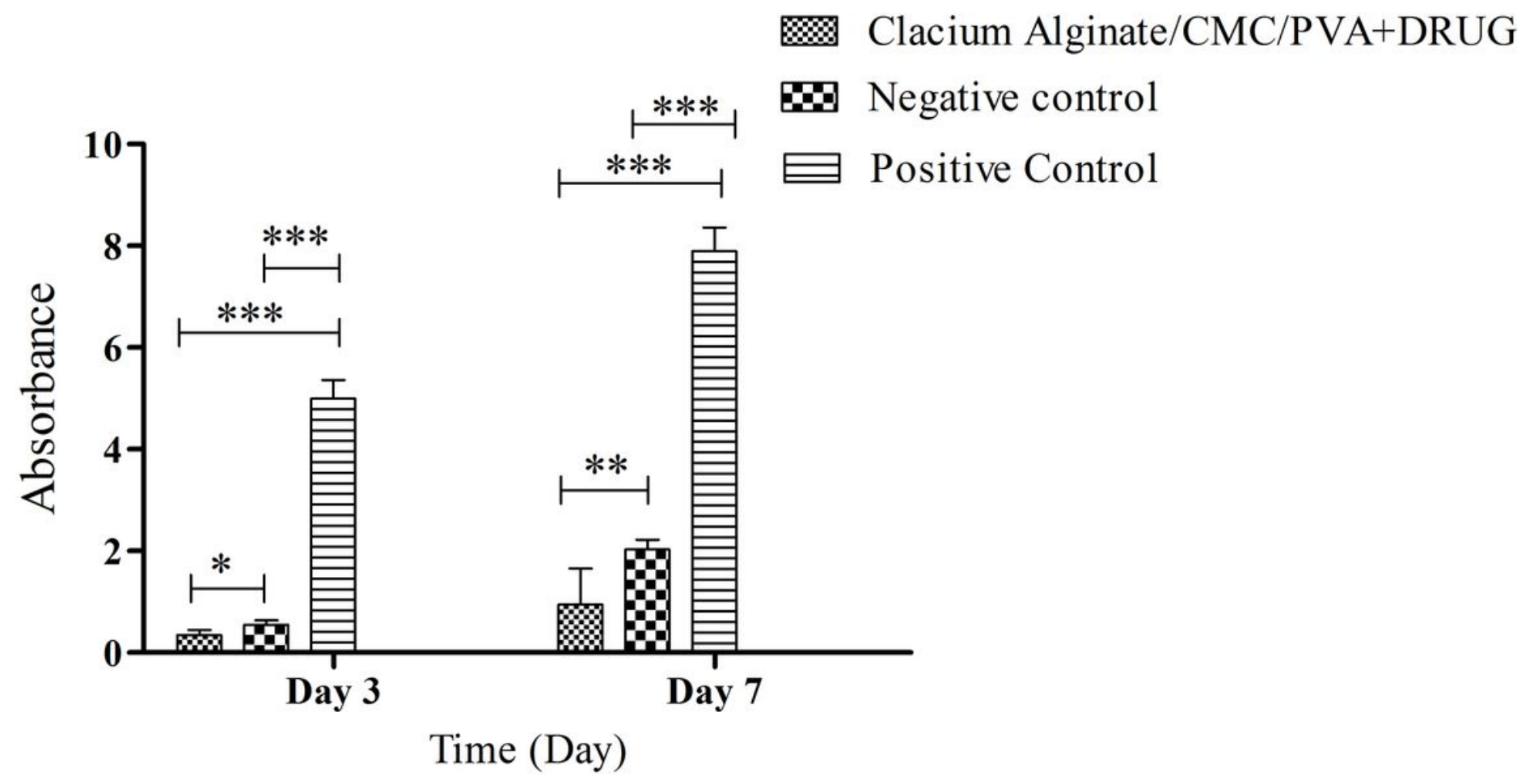

Figure 7

Microbial barrier property of the bilayer wound dressing after 3 and 7 days of incubation measured by Spectrophotometer at $600 \mathrm{~nm}$ 


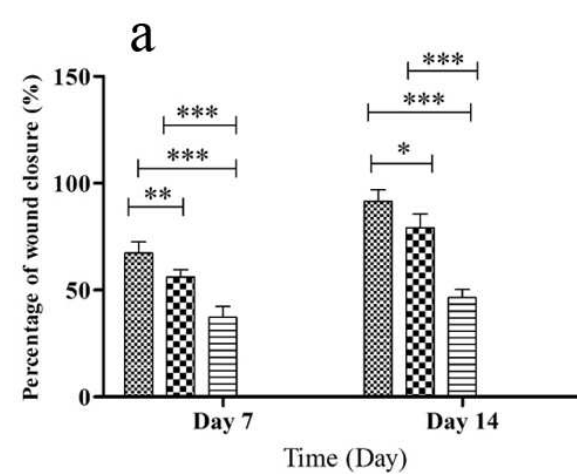

C

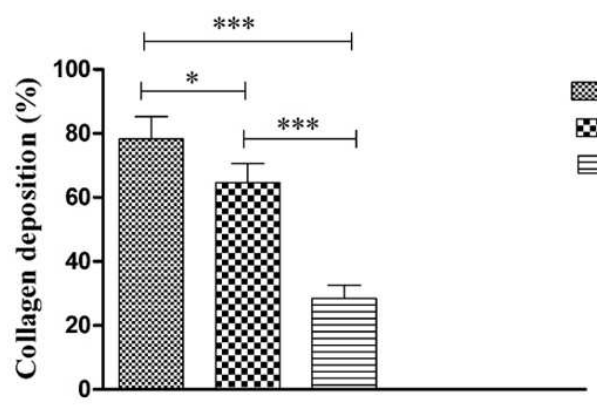

b

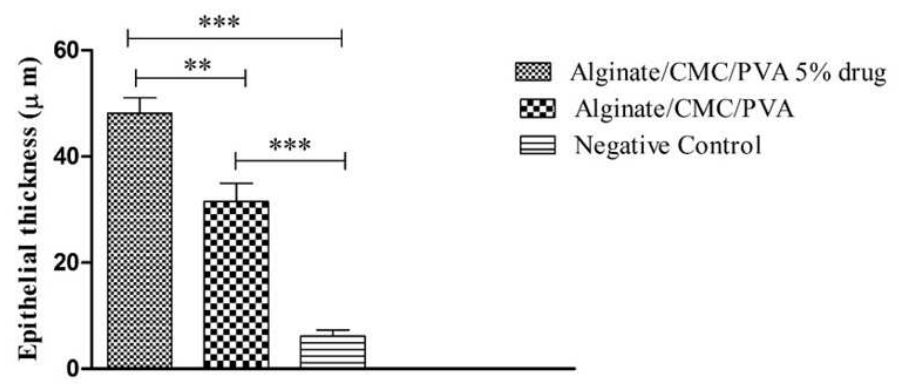

Alginate/CMC/PVA $5 \%$ drug

8 Alginate/CMC/PVA

Negative Control

\section{Figure 8}

Histogram comparing (a) percentage of wound closure at 7 days and 14 days post-wounding of different groups, (b) epithelial thickness of different experimental groups, and (c) the percentage of collagen deposition in different groups. Values represent the mean $\pm S D, n=5,{ }^{*}<<0.05,{ }^{\star} * P<0.01$ and ${ }^{\star \star \star} P<<0.005$ 
H\&E

\section{Drug loaded dressing}

\section{Drug free dressing}

\section{Negative control}

\section{Positive control}
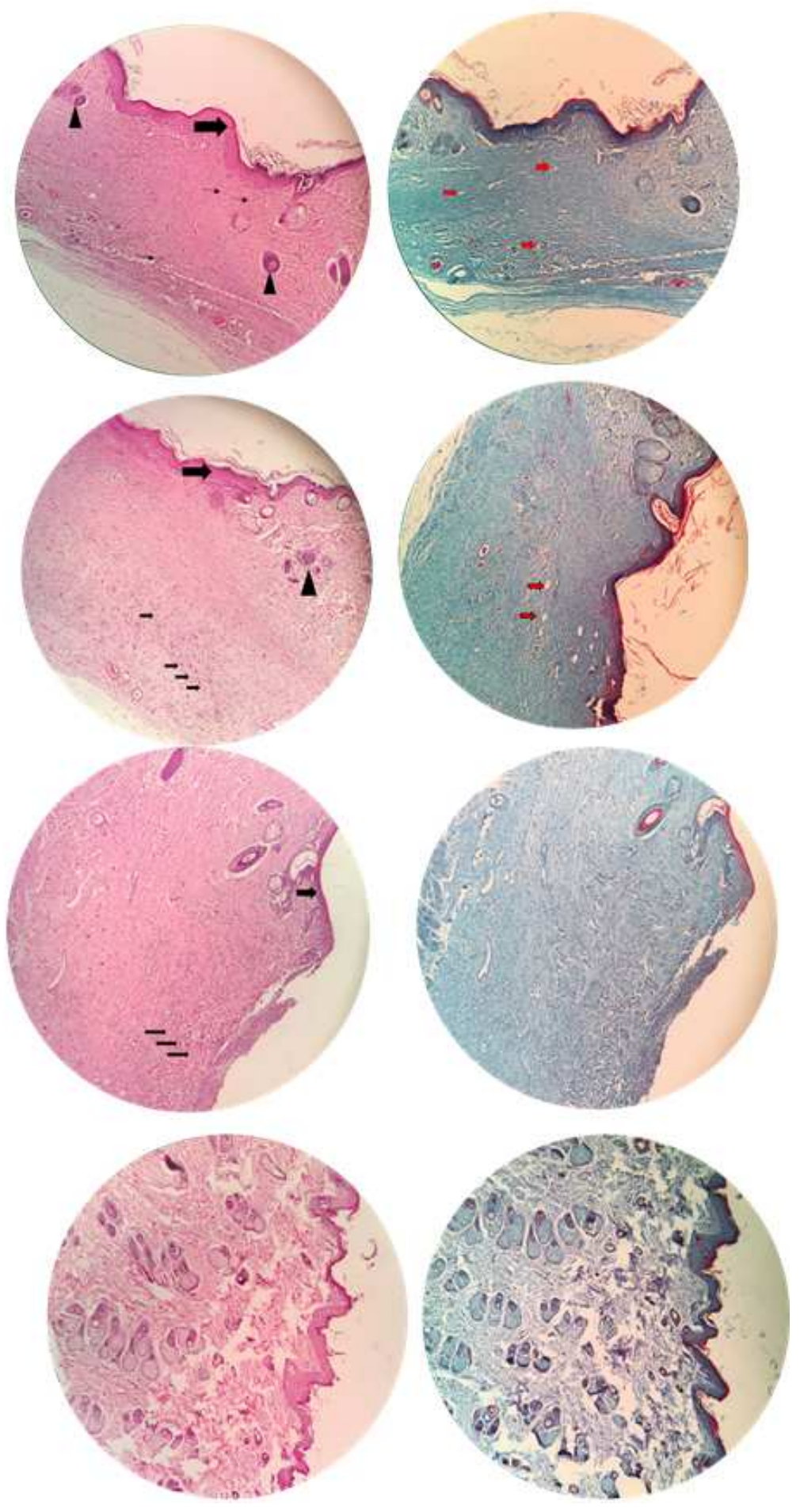

\section{Figure 9}

Hematoxylin and Eosin (H\&E) and Masson's trichrome (MT) stained microscopic sections (200x magnification) of wound tissues treated with different dressings 14 days' post-treatment. Thick arrows: epidermal thickness, thin arrows: recruitment of inflammatory cells, arrowheads: skin appendages, red arrows: neovascularization 